\title{
El voluntariado corporativo. Un modelo de responsabilidad empresarial para el desarrollo social
}

\author{
Maria Teresa GARCÍA NIETO*
}

Propuesto: 14 de marzo de 2012

Evaluado: 10 de abril de 2012

Aceptado: 5 de mayo de 2012

(Abstracts y palabras clave al final del texto)

\section{UNA CARRERA HACIA EL PROGRESO: LAS TEORÍAS DEL DESARROLLO}

La idea preliminar de "desarrollo", según las aportaciones de diferentes autores, fue expresada por el presidente de los Estados Unidos, Woodrow Wilson, en 19181. Sin embargo, se hizo necesario esperar a las postrimerías de la II Guerra Mundial, en un contexto geopolítico de descolonización y una situación económica protagonizada por el Plan Marshall, para que su referencia se generalizara. Las teorías clásicas de desarrollo se formulan con connotaciones definidas exclusivamente en el ámbito de la economía y la tecnología, y con el objetivo fundamental de lograr una mejora material. El desarrollo se vincula directamente con el progreso industrial. Las Teorías de la Modernización, del Tercer Mundo y de la Dependencia se encuentran en el marco de estas teorías clásicas del desarrollo.

Como reacción a estos supuestos economicistas y tecnológicos surgieron nuevos postulados de origen sociológico, nuevas teorías del desarrollo centradas en los sistemas sociales y en los factores culturales. La Teoría de los Sistemas Mundiales y la Teoría de la Globalización ofrecen nuevas perspectivas del desarrollo, entendiéndolo como un progreso no sólo económico o político, sino también y sobre todo, un progreso social y cultural.

\subsection{TEORÍA DE LA MODERNIZACIÓN}

Para la Teoría de la Modernización, el concepto de desarrollo ha servido para etiquetar a las sociedades en función del momento y el nivel de avance en esa progre-

\footnotetext{
* Universidad Complutense de Madrid

1 Discurso de los catorce puntos, del presidente Wilson al Congreso de los Estados Unidos, el 8 de enero de 1918.
} 
sión. Las colectividades más adelantadas son tomadas como arquetipo para las más demoradas en ese proceso. A las sociedades más avanzadas, las más productivas, se las considera desarrolladas mientras a aquellas no tan evolucionadas se las bautizaba con el calificativo de subdesarrolladas.

Walt Whitman Rostow ${ }^{2}$ construye un modelo de crecimiento económico, referencia obligada en las teorías del desarrollo. Rostow identifica el progreso económico con el proceso de modernización, y éste se caracteriza por ser:

- Homogeneizador, tendente a la convergencia entre las sociedades.

- Proclive a Europa y Norteamérica, a las que se considera dignas de ser imitadas por ser económicamente prósperas y políticamente estables.

- Inevitable e irreversible.

- Deseable.

- Un proceso de cambio a largo plazo, basado en la evolución. Un proceso no revolucionario (Reyes, p. 4).

Para Rostow una sociedad ha de evolucionar hacia la modernización y el desarrollo pasando por cinco etapas: una sociedad tradicional, la precondición para el despegue, el proceso de despegue, el camino hacia la madurez y una sociedad de alto consumo masivo. Así, cuando un país se sitúa en alguna de las etapas intermedias estaría en vías de desarrollo.

El desarrollo se identifica con el progreso y la civilización, el subdesarrollo, en cambio, se asocia con lo atrasado y con lo primitivo. El desarrollo se localiza en las naciones o estados más avanzados, en el Primer Mundo, y el subdesarrollo, por el contrario, se demarca en el denominado Tercer Mundo.

\subsection{TEORÍA DEL TERCER MUNDO}

La clasificación de los mundos como reflejo del nivel de desarrollo, en la que se establece la dicotomía entre el Tercer y el Primer Mundo, se la debemos al economista francés Alfred Sauvy (1952)3 ${ }^{3}$. Sauvy, tomando como referencia la idea del Tercer Estado, localiza tres mundos distintos: Primer Mundo, Segundo Mundo y Tercer Mundo. Estos términos no responden a un afán de orden, sino a la intención de distinguir y clasificar a los estados en función de estrictos criterios económicos. Así, se designa con el nombre de Primer Mundo al conjunto de estados capitalistas desarrollados; el Segundo Mundo se corresponde con el grupo de estados comunistas, en vías de desarrollo; y el Tercer Mundo estaría formado por el resto de los países, los llamador países "no alineados". El Tercer Mundo engloba a todos los países subdesarrollados, sufrientes de la desatención y de la explotación por parte de los otros dos mun-

2 Profesor de Historia americana en las Universidades anglosajonas de Oxford y Cambridge, de Historia de la Economía en el estadounidense Instituto Tecnológico de Massachusetts (MIT), y de Economía política en la Universidad de Texas.

3 Sauvy, A. (1952): “Troies Mondes, Une Planète”. L'Observateur, 14 août 1952, 118, p. 14. 
dos. El Tercer Mundo es el más importante y el más extenso y poblado, y se extiende por los continentes africano, asiático y en el centro y el sur de América. Curiosamente, esta clasificación fue adoptada como lábaro por algunos ideólogos de la izquierda, defensores del marxismo y del socialismo soviético. Esta división entre los países "explotadores del imperio capitalista del Norte, del Primer Mundo", y "los países explotados, marginados y sin posibilidad de desarrollo del Tercer Mundo", ofrece una visión fatalista del progreso de los estados, que durará prácticamente una década desde finales de los años 70 hasta la caída del Muro de Berlín en 1989.

\subsection{TEORÍA DE LA DEPENDENCIA}

Coincidiendo temporalmente con la formulación de Sauvy, surgen las bases de la "Teoría de la Dependencia", fruto de la contribución de varios analistas de la Comisión Económica para América Latina y el Caribe (CEPAL), órgano subordinado de la Organización de Naciones Unidas. El punto de partida de la Teoría de la Dependencia fue el modelo financiero propuesto por el economista argentino Raúl Prebisch, que sería reformulado posteriormente combinando elementos teóricos del neo-marxismo y del keynesianismo 4 . La Teoría de la Dependencia, focalizado en el concepto "centroperiferia", trata de explicar cómo el subdesarrollo y la pobreza de los países del Sur tienen su origen en las circunstancias históricas. Los países del Tercer Mundo, "de la periferia", han sido abastecedores de los recursos naturales y de la mano de obra barata de los países del Norte, "del centro", propiciando su enriquecimiento. Mientras, los países del Sur adquieren el stock de los encarecidos productos terminados que les ofrecen los países del Primer Mundo, perseverando de este modo en su penuria. De esta forma la relación de dependencia entre los países desarrollados del centro y los países subdesarrollados de la periferia se consolida y se perpetúa.

Para acabar con esta situación injusta de desequilibrio, la Teoría de la Dependencia señala la imperiosa necesidad de implementar, en los países subdesarrollados, políticas orientadas al autoabastecimiento, y a la vez establecer medidas comerciales proteccionistas en el mercado exterior, mediante impuestos y aranceles.

Las teorías mencionadas, la Teoría de la Modernización, la Teoría del Tercer Mundo y la Teoría de la Dependencia, plantean diversas propuestas ante estas situaciones de desequilibrio. La Teoría de la Modernización apuesta por la evolución progresiva de los países; la del Tercer Mundo conduce a la resignación y la Teoría de la Dependencia propugna drásticos cambios estructurales. Las propuestas, en todo caso, están basadas en parámetros exclusivamente económicos.

Giovanni E. Reyes ${ }^{5}$ presenta dos nuevas teorías sobre el desarrollo. Además de la Teoría de la Modernización y a la Teoría de la Dependencia, ya citadas, describe

4 Samir Amin, Enrique Cardoso, Theotonio Dos Santos, Andre Gunder Frank y Edelberto Torres-Rivas son algunos de los autores que protagonizaron la reformulación de la Teoría de la dependencia durante el segundo lustro de los años 50 y en la década siguiente.

5 Economista e ingeniero guatemalteco, doctor en Economía para el desarrollo y relaciones internacionales por la Universidad de Pittsburg, y ex-profesor de la Universidad Jesuita Centroamericana. 
con minuciosidad la llamada Teoría de los Sistemas Mundiales y la denominada Teoría de la Globalización, fundamentadas en aspectos sociales y culturales. Ambas teorías abandonan como unidades de estudio a las naciones-estados, para focalizar su análisis en los sistemas sociales.

\subsection{TEORÍA DE LOS SISTEMAS MUNDIALES}

Las nuevas circunstancias económicas de los años sesenta, del pasado siglo, dieron pié a un grupo de investigadores del Centro de Estudios de Economía, Sistemas Históricos y Civilización de la Universidad Estatal de Nueva York, encabezado por Immanuel Wallerstein ${ }^{6}$, a proponer una nueva teoría del desarrollo como reacción frente a los postulados de la Teoría de la Dependencia. La Teoría de los Sistemas Sociales, con origen en el ámbito de la Sociología, se extendió rápidamente a los dominios de la Historia, la Antropología, las Ciencias Políticas y el Urbanismo. Sus líneas de fuerza dejan de lado el tradicional concepto de naciones-estado y centran su discurso en los diversos sistemas que sustentan la sociedad, tanto dentro como fuera de un país. El proceso de desarrollo de los pueblos no es considerado perjudicial a priori, como afirma la Teoría de la Dependencia, aunque el grado de desarrollo puede avanzar o retroceder por diversas circunstancias. Tal teoría se preocupa, de una parte, por los sistemas vinculados a la investigación, además de por la aplicación y transferencia de tecnología; y por otra, por los sistemas relacionados con los temas financieros y del comercio exterior.

\subsection{TEORÍA DE LA GLOBALIZACIÓN}

Las condiciones políticas, económicas y sociales de un país, de acuerdo con la Teoría de la Globalización, están determinadas fundamentalmente por los factores culturales. Por ello, para estudiar los procesos de desarrollo es preciso analizar los vínculos culturales entre los países. Sobre todo, teniendo en cuenta la flexibilidad y la amplitud de los sistemas de comunicación forjados por las nuevas tecnologías de la información. Si la Teoría de los Sistemas Mundiales se centra en los sistemas sociales, la Teoría de la Globalización se centra en los sistemas de comunicación.

Moore, en su libro titulado Globalización y cambio social (1993), subraya el papel de la comunicación en la configuración de un mundo global interconectado. Los sistemas de comunicación propician el intercambio cultural al favorecer los procesos de interacción entre personas de diferentes poblaciones, y no sólo entre gobiernos e instituciones. Igualmente, con ello se favorece la integración de los grupos marginales. Asimismo, el acceso a los nuevos sistemas de comunicación, por parte de grandes y pequeñas empresas, ha generado un modelo de intercambio compartido que facilita y agiliza las transacciones comerciales.

6 Wallerstein: profesor de Sociología en la Universidad de Binghamton (SUNY). 
En definitiva, la Teoría de la Globalización, al igual que la ya descrita Teoría de la Modernización, define a Europa y a los Estados Unidos como paradigmas del desarrollo y las redes de comunicación contribuyen a la difusión de sus valores como arquetipo cultural dominante.

\section{UNA CONCEPCIÓN HUMANISTA DEL DESARROLLO}

Nuestra personal toma de posición sobre estos temas nos sitúa dentro de las teorías evolucionadas y menos economicistas del desarrollo, defendiendo así, una perspectiva más humanista. Para lograr el desarrollo de un país es necesario el progreso económico, pero éste debe ir acompañado del ineludible progreso social y del desarrollo humano. Desde esta postura intelectual, el concepto de "desarrollo" se puede definir como la evolución de las comunidades o grupos sociales hacia los niveles óptimos del bienestar de sus integrantes. Y, aunque es innegable que el avance económico y tecnológico juega un papel relevante en el desarrollo de los pueblos, estos factores no son ni los únicos, ni los más importantes coadyuvantes del progreso social. La integración de los derechos de las personas, como la salud, la educación o la vivienda digna, se suman a la idea de desarrollo. Podemos afirmar, por tanto, que el desarrollo es un proceso de cambio y mejora de las condiciones de vida de las personas que conforman una colectividad, independientemente de su raza, sexo, edad, localización geográfica o cualquier otra variable que pueda identificarla.

La Ley española 23/1998, de 7 de julio, de Cooperación Internacional para el Desarrollo, adopta la doctrina humanista al fundamentarse en los siguientes principios:

a) El reconocimiento del ser humano en su dimensión individual y colectiva, como protagonista y destinatario último de la política de cooperación para el desarrollo.

b) La defensa y promoción de los Derechos humanos y las libertades fundamentales, la paz, la democracia y la participación ciudadana en condiciones de igualdad para mujeres y hombres y, en general, la no discriminación por razón de sexo, raza, cultura o religión y el respeto a la diversidad.

c) La necesidad de promover un desarrollo humano global, interdependiente, participativo, sostenible y con equidad de género en todas las naciones, procurando la aplicación del principio de corresponsabilidad entre los Estados, en orden a asegurar y potenciar la eficacia y coherencia de las políticas de cooperación al desarrollo en su objetivo de erradicar la pobreza en el mundo.

d) La promoción de un crecimiento económico duradero y sostenible de los países, acompañada de medidas que promuevan una redistribución equitativa de la riqueza, para favorecer la mejora de las condiciones de vida y el acceso a los servicios sanitarios, educativos y culturales, así como el bienestar de sus poblaciones.

e) El respeto a los compromisos adoptados en el seno de los organismos internacionales. 
Los 193 países de la Organización de las Naciones Unidas acordaron, en el año 2000, alcanzar en un plazo de 15 años, los llamados "Objetivos de Desarrollo del Milenio". Estos objetivos, son considerados desde entonces, como criterio de mínimos para el desarrollo de las diferentes comunidades en todo el mundo 7 :

- Erradicar la pobreza extrema y el hambre.

- Lograr la enseñanza primaria universal.

- Promover la igualdad de género y el empoderamiento de las mujeres.

- Reducir la mortalidad de los niños y niñas menores de cinco años.

- Mejorar la salud materna.

- Combatir el VIH/SIDA, el paludismo y otras enfermedades.

- Garantizar la sostenibilidad del medio ambiente.

- Fomentar una alianza mundial para el desarrollo.

Siguiendo este planteamiento, el Programa de las Naciones Unidas para el Desarrollo (PNUD) propone, como indicador del desarrollo de cada país, el llamado "índice de desarrollo humano". Este índice, indicador social resumen de los atributos de una sociedad, se determina por tres parámetros:

- Salud, esperanza de vida.

- Educación, alfabetización y nivel de estudios.

- Nivel de vida digno, Producto Interior Bruto (PIB) per cápita.

Resulta coherente que la Organización de las Naciones Unidas tome como unidad de análisis a los países. Sin embargo, desde el planteamiento humanista no es exactamente así. El nivel de desarrollo constituye una variable comparativa que sirve también para identificar el progreso social de diferentes comunidades, grupos o colectivos humanos, estén localizadas fuera o dentro de un ámbito geográfico concreto. Así pues, la posibilidad de contribuir a la mejora de las condiciones de vida de un grupo social "subdesarrollado" se hace más sencilla y evidente cuanto más próximo a nosotros se encuentre. De este modo, no sólo las organizaciones internacionales o los estados han de ser considerados agentes para el cambio social; no sólo son las administraciones públicas las únicas responsables. El llamado tercer sector, formado por las entidades sin ánimo de lucro como las organizaciones no gubernamentales o las fundaciones, también tiene un compromiso claro y explícito con el desarrollo. Y las empresas y corporaciones han de asumir, asimismo, sus obligaciones sociales mediante el ejercicio de la responsabilidad social empresarial o corporativa (RSE / RSC).

\section{LA EMPRESA COMO AGENTE DE DESARROLLO SOCIAL.}

El siglo XX ha consolidado el papel fundamental de las organizaciones empresariales en el "desarrollo económico" de una comunidad. Pero, además, hoy nadie

7 Naciones Unidas (2010):“Objetivos de desarrollo del Milenio” Informe 2010. New York. 
cuestiona el añadido protagonismo de las corporaciones como entidades de "desarrollo social". Un protagonismo alcanzado en las últimas dos décadas del pasado siglo y afianzado durante los primeros lustros del actual, explicitado en el ejercicio de la responsabilidad social empresarial (RSE).

En los llamados países desarrollados ha crecido de forma exponencial el número de organizaciones que han adoptado, en sus planes estratégicos, políticas de responsabilidad social corporativa. España no ha sido una excepción, si bien es cierto que se aprecia sobre todo en el ámbito de las grandes compañías. Según el Informe Forética $^{8} 2011$ sobre la evolución de la responsabilidad social de las empresas en España, el 97\% de las grandes compañías conoce la RSE, y cuatro de cada diez desarrollan planes específicos en esta materia, aunque sólo el 5\% los haya formalizado por escrito. Sin embargo, en el caso de las organizaciones con menos de cien empleados, casi la mitad de ellas todavía no ha oído hablar de la RSE.

La concienciación de los empresarios y directivos ha constituido, sin duda alguna, la pieza clave para el emprendimiento de estos programas de responsabilidad social empresarial. De acuerdo con los datos del mencionado informe, en siete de cada diez empresas los planes de RSE son formulados directamente por el consejo de administración o por la dirección general de la empresa.

Pero la responsabilidad social de las empresas había sido ya reclamada décadas antes desde los postulados científicos, tanto desde las doctrinas de la gestión empresarial, como desde los planteamientos teóricos de las de las relaciones con los públicos, también conocidas como teorías de los stakeholders o de los públicos estratégicos (García Nieto, 2012). Las premisas de la gerencia de negocios reivindicaban la RSE como parte integrante de la estrategia empresarial con el fin de optimizar la eficacia las organizaciones. En las teorías de las relaciones con los públicos, la RSE resulta esencial en la planificación del comportamiento corporativo, para alcanzar el objetivo de mejorar esas relaciones, así como de lograr el mutuo entendimiento entre la organización y sus públicos.

El abogado vienés Meter Ferdinand Drucker, formado en los principios de la economía de la mano del británico Keynes, propone a finales de los años sesenta del pasado siglo, una teoría sobre la gerencia y la administración de las empresas que transformaría de forma radical el mundo de los negocios. Sobre los pilares de conceptos como la dirección por objetivos, la descentralización, el emprendimiento y la innovación, junto con las nuevas tecnologías y la evolución desde la sociedad de la información hacia la sociedad del conocimiento, Drucker entiende la responsabilidad social no como una mera declaración de buenas intenciones, sino como parte de la estrategia del propio negocio (Drucker, 1968). Al tiempo, considera imprescindible integrar, dentro de la gestión empresarial, las políticas de desarrollo social dirigidas a satisfacer las necesidades y los intereses de los grupos sociales con los que se relaciona la empresa (1984).

El citado letrado enuncia dos modos de concebir la responsabilidad social de las empresas. El primero consiste en impedir, minimizar o poner fin a todo posible

\footnotetext{
8 Forética es una asociación de empresas y profesionales implicados en el desarrollo de la responsabilidad social corporativa en España, nacida en 1999, cuenta hoy con más de 140 organizaciones asociadas y más de 100 socios personales.
} 
impacto nocivo que la propia existencia de la empresa o sus actividades pudieran ocasionar a la sociedad. El segundo, trata de solucionar los problemas sociales, que deben ser percibidos por la empresa como oportunidades.

Si con Drucker la responsabilidad social empresarial adquiere naturaleza gerencial al convertirse en parte integrante e indisoluble de la gestión estratégica de las empresas, con Archie B. Carroll ${ }^{9}$ la responsabilidad social se convierte en el fundamento de la gestión misma, entendida como respuesta a un imperativo de sostenibilidad. Es un requerimiento de los procesos de toma de decisiones en las empresas para asegurar su adecuación a unos objetivos de equilibrio y a la salvaguarda de los intereses de los diferentes públicos implicados (Carroll y Buchholtz, 2011). Para este autor la responsabilidad social empresarial incluye cuatro categorías representadas gráficamente a modo de pirámide. En la base se halla la responsabilidad económica; en el siguiente nivel está la responsabilidad legal; sobre ella se localiza la responsabilidad ética y en el nivel superior está la responsabilidad filantrópica o discrecional, de carácter voluntario (Carroll, 1991, 1999).

Definamos los conceptos. La responsabilidad económica, deriva de la naturaleza productiva de las empresas y se traduce en tres compromisos: En primer lugar, el de conseguir la máxima rentabilidad y los mayores beneficios; en segundo término, optimizar la calidad de los productos y en tercer lugar, garantizar las mejores condiciones laborales de los empleados. La responsabilidad legal, por su parte, impone a la organización el deber de cumplir las normas jurídicas reguladoras de la actividad empresarial como aval del desarrollo social. La responsabilidad ética impone a la organización la necesidad de adaptar su comportamiento a las normas sociales acordes con el criterio de lo justo y lo correcto. De tal manera que la organización se rija por los valores de justicia, respeto y equidad. La responsabilidad filantrópica, asumida de forma voluntaria por la organización, consiste en la aplicación de políticas orientadas a la satisfacción de los intereses sociales como clara contribución al de-sarrollo social de una comunidad.

James Grunig ${ }^{10}$, encabezando a un grupo de investigadores de las relaciones públicas, nos ofrece otra clasificación de la responsabilidad social empresarial $(1984,1992)$, en la que se describen tres niveles de compromisos a cumplir por las organizaciones: los compromisos primarios, los secundarios y los sociales.

Los compromisos primarios se corresponden con las funciones básicas de la actividad productiva de la empresa. La creación de puestos de trabajo y la fabricación de productos de calidad, estarían en este primer nivel de responsabilidad. Los compromisos secundarios buscan evitar y paliar posibles consecuencias negativas del desarrollo de las actividades empresariales sobre los diferentes públicos. En este sentido, las empresas están obligadas, por ejemplo, a acabar con los efectos contaminantes de su actividad. Los compromisos sociales atañen a la implementación de políticas sociales ajenas a las funciones propias de la organización. Se trata de poner en marcha planes de acción dirigidos a la satisfacción de los intereses y de las necesidades de diversas personas o grupos sociales. Por ejemplo, las

9 Profesor emérito del Departamento de Gestión de la Escuela de Negocios Terry en la Universidad estadounidense de Georgia.

10 Profesor emérito de Comunicación de la Universidad norteamericana de Maryland. 
contribuciones para la mejora de su calidad de vida y las ayudas a la comunidad en las formas más diversas.

Grunig llama "responsabilidad social" a los compromisos sociales, porque afectan a personas no necesariamente relacionadas con la empresa; a los otros dos compromisos los nombra de "responsabilidad pública", por incumbir a personas vinculadas con la empresa. No obstante, estas denominaciones no parecen ser las más adecuadas. Cuando hablamos de públicos hablamos de grupos sociales tipificados y por ende, los tres niveles de responsabilidad descritos por este autor se corresponden, en realidad, con formas distintas de interpretar la responsabilidad social.

El carácter discrecional y voluntario, y la finalidad de satisfacer los intereses sociales propiciando el desarrollo social, se presentan como rasgos comunes a la "responsabilidad filantrópica" de Carroll y al tercer nivel de responsabilidad de Grunig. Por ese motivo, nosotros nos referirnos a estos modos de ejercer la responsabilidad como "responsabilidad solidaria".

En cualquier caso, es preciso señalar que el ejercicio de la responsabilidad solidaria por parte de una organización puede plantearse con una doble finalidad:

- un objetivo social: satisfacer las necesidades y los intereses de determinados grupos sociales.

- un objetivo persuasivo: crear una imagen pública de la organización como empresa socialmente responsable, para mejorar su aceptación y lograr el apoyo por parte de los diferentes públicos. En este caso habrá un claro desequilibrio entre el esfuerzo social de la empresa y sus inversiones comunicativas, dando prioridad a éstas últimas.

Como advertía Ban Ki-moon, Secretario General de las Naciones Unidas, en la cumbre de negocios celebrada en Nueva York el catorce de febrero de 2012, es preciso reforzar los incentivos al ejercicio de la RSE, ya que muchas empresas limitan sus esfuerzos de sostenibilidad a programas piloto que nunca se terminan. O lo que es peor, la RSE se vuelve más una cuestión de imagen que una forma de operar. Es necesario que los principios de la sostenibilidad se incorporen de verdad a la cultura empresarial. En este sentido se encuentran iniciativas de la ONU como la del "Pacto Mundial". En este foro, que agrupa a casi 7.000 compañías en 140 países, las empresas se comprometen a alinear sus estrategias con los derechos humanos, los estándares laborales, el medio ambiente y la anticorrupción ${ }^{11}$.

La responsabilidad solidaria no puede ser sólo una cuestión de imagen y, por tanto, no puede concebirse únicamente como una herramienta persuasiva más. La responsabilidad solidaria implica a la propia filosofía gerencial de las organizaciones, y como tal ha de planificarse estratégicamente para alcanzar los objetivos de desarrollo social sostenible.

$11 \mathrm{http}: / /$ comunicarseweb.com.ar/?Ban_Ki-moon_alerto_que_la_sustentabilidad_no_puede_ser_una

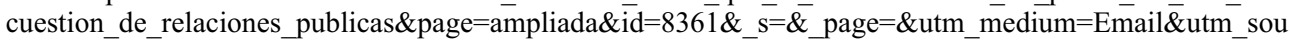
rce $=$ Newsmaker\&utm_campaign=Newsmaker-onu-rse-rrpp-23-02-2012. 


\section{EL VOLUNTARIADO CORPORATIVO, UN EJERCICIO DE RESPONABILIDAD SOLIDARIA PARA EL DESARROLLO}

El voluntariado corporativo, también llamado de empresa, debe ser entendido como una pieza del plan de responsabilidad solidaria de una organización, y éste, a su vez, como ya hemos señalado, ha de ser parte del plan estratégico de la gestión empresarial.

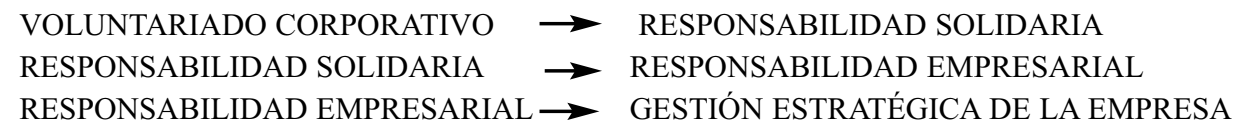

En los programas de voluntariado empresarial los empleados y las empleadas son los protagonistas. Éstos al implementar los proyectos de RSE, encarnan el compromiso social de la organización. Sin embargo, si bien es el personal de la empresa el encargado de la ejecución de las acciones solidarias, es la organización la que, al integrarlas en sus estrategias sociales, debe ocuparse de su planificación, supervisión y evaluación. Además la organización ha de demostrar su autoridad y su liderazgo social motivando y fomentando la implicación de su capital intelectual en dichos proyectos. Siempre, por supuesto, de forma coordinada y con la participación de los propios trabajadores en los procesos de toma de decisiones.

En la mayoría de los casos de voluntariado corporativo, la empresa lo planifica en coordinación con una organización no lucrativa (ONL) de tal modo que permita optimizar la eficacia de los proyectos sociales. Ello, requiere una definición muy precisa de los objetivos y de las acciones, con una demarcación temporal, económica, geográfica y social, nítida. De este modo, la ONL encauza los recursos y el trabajo solidario de la empresa para el logro de unas metas muy concretas de desarrollo social.

Debe quedar claro que en la práctica del voluntariado corporativo, los voluntarios forman parte del personal de la organización empresarial con la que mantienen una relación contractual remunerada y que, en ningún caso estas personas podrán recibir contraprestación económica alguna por su participación como voluntarias. El voluntariado corporativo ha de regirse por la normativa vigente. La Ley Estatal 6/1996 de Voluntariado entiende por tal al conjunto de actividades de interés general, desarrolladas por personas físicas, siempre que las mismas no se realicen en virtud de una relación laboral, funcionarial, mercantil o cualquier otra retribuida. Se entienden por actividades de interés general las asistenciales, de servicios sociales, cívicas, educativas, culturales, científicas, deportivas, sanitarias, de cooperación al desarrollo, de defensa del medio ambiente, de defensa de la economía o de la investigación, de desarrollo de la vida asociativa, de promoción del voluntariado, o cualesquiera otras de naturaleza análoga.

Además, la Ley exige una serie de requisitos para la práctica del voluntariado:

a) Que tengan carácter altruista y solidario.

b) Que su realización sea libre, sin que tengan su causa en una obligación personal o deber jurídico. 
c) Que se lleven a cabo sin contraprestación económica, sin perjuicio del derecho al reembolso de los gastos que el desempeño de la actividad voluntaria ocasione.

d) Que se desarrollen a través de organizaciones privadas o públicas y con arreglo a programas o proyectos concretos.

Quedan, así, excluidas de la condición del voluntariado social las actuaciones aisladas, esporádicas o prestadas al margen de organizaciones públicas o privadas sin ánimo de lucro, ejecutadas por razones familiares, de amistad o buena vecindad.

Pero, como ya se ha señalado, para garantizar la eficacia de los programas de voluntariado corporativo es necesaria su planificación, seguimiento y evaluación por parte de la empresa. Forética, responsable de la implantación en España de los Premios Europeos de Voluntariado Corporativo, indica siete claves como condicionantes del éxito de los programas de voluntariado empresarial ${ }^{12}$.

a. Desarrollar el Plan de Voluntariado Corporativo a partir de la estrategia corporativa de RSC.

b. Crear una comunidad virtual de empleados interesados en formar parte del programa de voluntariado corporativo, y dotarla de herramientas básicas de comunicación en red y de reporting de actividades (Portal de Voluntariado).

c. Contactar con entidades seleccionadas para identificar proyectos de interés común y cerrar acuerdos estratégicos o tácticos de colaboración.

d. Crear una organización virtual de gestión del programa, formada por empleados voluntarios, y coordinada desde la dirección.

e. Analizar, seleccionar y desarrollar propuestas de proyectos y tareas de voluntariado (propuestos por la empresa o por los empleados).

f. Benchmarking, evaluación comparativa, e intercambio de experiencias con empresas afines.

g. Registrar, visibilizar y reconocer la participación de los empleados en los proyectos de acción social apoyados por la empresa.

Sin embargo, hemos de decir que el voluntariado corporativo no sólo constituye un ejercicio de la responsabilidad social de la empresa. Es también un instrumento fundamental para la mejora de las relaciones entre la empresa y sus públicos internos:

— Promoviendo la identificación de los trabajadores con la cultura corporativa de la empresa.

- Favoreciendo la motivación laboral de los trabajadores.

- Acrecentando en ellos el sentimiento de integración y de pertenencia a la compañía.

- Propiciando la cohesión social interna en la empresa.

- Como forma de reconocimiento personal de los trabajadores por parte de la organización.

12 Paloma Lemonche (2011): Voluntariado Corporativo. Un puente de colaboración entre la empresa y la sociedad. Madrid: Forética. 
— Fomentando su formación.

- Facilitando la participación de los trabajadores en los procesos de toma de decisiones.

Según el Informe 2010 del Observatorio de Voluntariado Corporativo ${ }^{13}$, en algunas compañías los programas de voluntariado empresarial se plantean también con el objetivo de optimizar el entendimiento mutuo entre la organización y sus empleados. Cuatro de cada diez entidades consideran que el voluntariado corporativo sirve para fomentar el orgullo de pertenencia de los empleados a la empresa. Tres de cada diez lo estiman positivo para mejorar el clima laboral de la organización. Y una de cada cinco entiende que el voluntariado corporativo favorece el desarrollo de las capacidades de los empleados.

Estos datos muestran que todavía queda mucho por hacer. Se debe integrar el voluntariado corporativo como estrategia socialmente responsable en la gestión empresarial para el desarrollo. El voluntariado corporativo ha de plantearse como un elemento fundamental de las relaciones que la organización mantiene con sus públi$\cos$, tratando de satisfacer las necesidades y los intereses no sólo de los colectivos que reciben la ayuda prestada por los empleados voluntarios, sino también de éstos como públicos internos de la organización.

\section{UNA TIPOLOGÍA DEL VOLUNTARIADO EMPRESARIAL}

Para establecer el programa más adecuado para cada empresa es preciso tener en cuenta las diferentes variables que entran en juego en el ejercicio del voluntariado en relación con su capital intelectual. Siguiendo el citado cuaderno de Forética sobre voluntariado corporativo es preciso valorar:

a. La capacitación del personal voluntario. Hay que comprobar si se necesita o no una preparación especial previa, o si se requiere voluntarios experimentados y profesionales.

b. El destino de las personas voluntarias. Hay que considerar si es preciso desplazarse a los lugares donde se desarrollen los programas de voluntariado, ya sean acciones locales próximas a los lugares de residencia o de trabajo, o trabajos de cooperación al desarrollo que supongan viajar a otros países.

c. Los motivos y las áreas de actuación. Dependiendo de que sean acciones de asistencia social y de salud, de educación o formación y de capacitación e integración laboral, de preservación del medio ambiente y de protección del patrimonio cultural, de cooperación al desarrollo y de emergencia humanitaria.

d. Las funciones a desempeñar. Tan variadas como los proyectos de acción social, desde la captación de fondos y recursos o la organización de campañas

13 Observatorio de Voluntariado Corporativo (2010): Voluntariado Corporativo en España. Cooperación Internacional ONG e IESE Business School. Informe diciembre 2010. 
y eventos, hasta la realización de acciones de desarrollo y emergencia o las actividades de asistencia y formación.

e. El tiempo a emplear en actividades de voluntariado. Lo más habitual es la cesión de dos horas de trabajo al mes por empleado para dedicarlas al voluntariado corporativo o su equivalencia en días, veintidós horas al año o tres días.

Y atendiendo a estas consideraciones, los gestores empresariales deben definir y planificar sus programas de voluntariado corporativo decidiendo entre diferentes modalidades:

- Donaciones de fondos (matching giving) hechas por los empleados para proyectos sociales. La donación habrá de ser gestionada por la empresa teniendo en cuenta sus implicaciones económicas, legales y fiscales.

- La empresa canaliza las iniciativas de voluntariado de sus empleados. La compañía organiza unidades de trabajo para el desarrollo de proyectos de acción social propuestos por los propios empleados, en colaboración con una organización no lucrativa. En esta modalidad, la empresa actúa como coordinadora del voluntariado (matching individual).

- Microvoluntariado virtual. Los empleados de una empresa desarrollan desde sus puestos de trabajo, pequeñas tareas para una ONG. El empleado utiliza las infraestructuras tecnológicas de su empresa para colaborar con una ONG con actividades que duran entre 15 minutos y dos horas. Vehicularlo a través del portal de voluntariado disponible en la intranet corporativa ofrece numerosas ventajas a la empresa a la hora de evaluar su eficacia.

- Microdonaciones de empleados (teaming). Se trata de donaciones puntuales o periódicas de un euro o céntimos de euro, realizadas por los empleados o miembros de cualquier organización para el desarrollo de una acción social decidida democráticamente por los propios donantes. La donación de los empleados, que no tienen implicaciones fiscales dada su reducida cuantía, suele complementarse con una cantidad total equivalente aportada por la misma empresa.

- Campañas. Consiste en la participación de los empleados de la organización en acciones ocasionales de duración limitada y con un fin específico, generalmente determinadas con antelación por la dirección de la empresa.

- Bancos de tiempo. Los empleados dedican parte de su horario laboral a actividades de voluntariado, de acuerdo con una cuota temporal concedida por la empresa para este fin, que suele oscilar desde dos horas al mes hasta una hora a la semana.

- Outdoor solidario. Consiste en el desarrollo de acciones por parte de los empleados, fuera de las instalaciones de la empresa, en las que se combinan los objetivos sociales o medioambientales y el carácter lúdico. Estas actividades generan espíritu de equipo y cohesionan el grupo. Algunos ejemplos son la limpieza de bosques, la visita a hospitales o la formación a colectivos marginales. 
- Voluntariado profesional. Los empleados voluntarios prestan un servicio profesional a una ONG sin coste o con un precio simbólico. Es lo que se llama servicios "pro-bono".

- Cesión de capital humano. La empresa cede su capital intelectual, personas cualificadas, a una ONG durante un periodo determinado.

A modo de conclusión, podemos afirmar cómo el voluntariado corporativo es una práctica cada vez más extendida en las grandes empresas españolas. El mencionado informe 2010 del Observatorio de Voluntariado Corporativo indica que en sesenta y ocho empresas de las ciento cinco organizaciones estudiadas, con más de quinientos empleados, se realizan prácticas de voluntariado corporativo. Las compañías optan sobre todo por programas de voluntariado de intervención social (74\%), seguidos a mucha distancia del voluntariado medioambiental y del profesional (34\% y $22 \%$ respectivamente). Si bien es cierto que este dato no es ni mucho menos generalizable ni extrapolable al resto del censo empresarial, sí puede ser tomado como un indicador de la tendencia de las grandes empresas y servir de ejemplo para el sector empresarial español.

Los empresarios españoles tienen que concienciarse cada vez más del importante papel de sus compañías como agentes promotores del desarrollo social sostenible. Han de asumir la necesidad de integrar programas de responsabilidad solidaria en sus planes de gestión empresarial. Y deben acostumbrarse a incorporar el voluntariado corporativo como una práctica habitual en las estrategias de entendimiento mutuo entre la organización y sus públicos internos. En ello confiamos todos. 


\section{REFERENCIAS BIBLIOGRÁFICAS}

CArroll, A.B. (1991): "The Pyramid of Social Responsibility: Toward the Moral Management of Organizational Stakeholders". Business Horizons, july-august, pp. 3948.

(1999): "Corporate social responsibility: evolution of a definitional construct". Business and Society, vol. 38, n 3, pp. 268-295.

Carroll, A.B. Y BuchHoltz, A.K. (2011): Business and Society: Ethics, Sustainability, and Stakeholder Management, 8th Edition. Boston: South-Western College Publishing.

Dos Santos, T. (1971): La Estructura de la Dependencia. Boston: Ed. Extending Horizons.

Drucker, P.F. (1968): Drucker, Frontiers of Management. Nueva York, Truman Talley Libros.

- (1984): "The New Meaning of Corporate Social Responsibility" En California Management Review. Vol. 40, Issue: 2, pp. 8-17.

- (1984): "Una nueva mirada a la Responsabilidad Social Corporativa", McKinsey Quarterly, 1984.

FORÉTICA (2011): 1 Informe Forética 2011 sobre la evolución de la responsabilidad social de las empresas en España. Madrid: Forética.

GARcíA Nieto, M.T. (2012): "Las ciencias sociales y la responsabilidad social corporativa". ADResearch ESIC. Num. 6. Julio 2012. Madrid: ESIC.

Gutierrez R., R. (2004): "Walt W. Rostow: réquiem por un historiador económico". Ciencia ErgoSum. Vol 10, Num. 003. Nov 2003-Febr 2004. Toluca, México, pp. 295-303.

Grunig, J.E. \& Hunt, T. (1984): Managing Public Relations. New York: Holt, Rinehart and Winston.

GRUNIG, J.E. (Ed.) (1992): Excellence in Public Relations and Communication Management: Contribution to Effective Organizations. Hillsdale, New Jersey: Lawrence Erlbaum Associates.

LEMONCHE, P. (2011): Voluntariado Corporativo. Un puente de colaboración entre la empresa y la sociedad. Madrid: Forética.

LEY 6/1996, de 15 de enero, del Voluntariado.

LEY 23/1998, de 7 de julio, de Cooperación Internacional para el Desarrollo.

Ministerio de SANIDAD y Política Social (2009): Normativa Española sobre Voluntariado. Madrid: MSPS. 5. ${ }^{\mathrm{a}}$ ed.

MoOre, M. (1993): Globalization and social change. New York: Elseiver.

ObSERVATORIO DE Voluntariado Corporativo (2010): Voluntariado Corporativo en España. Cooperación Internacional ONG e IESE Business School. Diciembre 2010.

ONU (2010): Objetivos de desarrollo del Milenio. Informe 2010. New York: Naciones Unidas.

Prebisch, R. (1950): The Economic Development of Latin America and Its Principal Problems. New York: United Nations.

REYES, G. E. (2001): “Teoría de la Globalización: bases fundamentale”. Revista Tendencias Vol. II,.1, junio: 43-53.

(2002): Principales teorías sobre desarrollo económico y social y su aplicación en América Latina y el Caribe. www.zonaeconomica.com.

Rostow, W.W. (1960): "The Stages of Economic Growth: A Non-Communist Manifiesto". Londres: Cambridge University Press.

Sauvy, A. (1952): “Troies Mondes, Une Planète”. L'Observateur, 14 août 1952, n¹18.

Wallerstein, I. (1987): World-System Analysis. Standford: Standford University Press. 


\title{
RESUMEN
}

El voluntariado empresarial, denominado también corporativo, constituye la mejor manifestación del ejercicio de la responsabilidad social de las organizaciones al poner en práctica sus programas de acción social para el desarrollo. La colaboración voluntaria y libre de las personas vinculadas laboralmente a una organización, realizando actividades destinadas a la mejora del bienestar de otras personas o grupos sociales, debe responder a una adecuada planificación con el objetivo de optimizar la eficacia de sus esfuerzos. Al mismo tiempo, el voluntariado empresarial es un instrumento fundamental para la mejora de las relaciones entre la empresa y sus públicos internos, propiciando la integración, la cohesión y la motivación de sus empleados, y como tal debe ser estratégicamente diseñado para el cumplimiento de estos fines.

Palabras clave: Voluntariado corporativo, voluntariado empresarial, responsabilidad social corporativa, responsabilidad social empresarial, relaciones con los públicos internos, ciudadanía corporativa, empresa solidaria, comunicación para el desarrollo.

\begin{abstract}
Corporate or business volunteerism is the best manifestation of the exercise of the social responsibility in the organizations when they want to implement its social action programs for the development. Free and voluntary collaboration of the employees of an organization, performing activities aimed at improving the welfare of other people or social groups must respond to a proper planning in order to optimize the effectiveness of their efforts. At the same time, volunteering is a key business for the improvement of relations between the company and its internal stakeholders, promoting integration, cohesion and motivation of its employees, and so it should be strategically designed to achieve these purposes.
\end{abstract}

Key words: Corporate volunteering, business volunteering, corporate social responsibility, business social responsibility, internal public relations, corporate citizenship, solidarity enterprise, communication for development.

\section{RÉSUMÉ}

L'entreprise volontaire, appelée également entreprise est la meilleure manifestation de l'exercice de la responsabilité sociale des organisations, de mettre en pratique leur action sociale pour les programmes de développement. Les volontaires de collaboration gratuits sont des personnes professionnellement liées à une organisation, qui mènent des activités visant à l'amélioration du bien-être des autres personnes ou de groupes sociaux, et dont la contratation doit répondre à une planification appropriée dans le but d'optimiser l'efficacité de leurs efforts. Au même temps, le bénévolat corporatif est un instrument essentiel pour l'amélioration des relations entre la compagnie et son public interne, pour favoriser l'intégration, la cohésion et la motivation de ses employés et comme telle, il doit être stratégiquement conçu pour ces fins.

Mots clé: Bénévolat corporatif, bénévolat corporatif, responsabilité sociale des entreprises, responsabilité sociale des entreprises, relations internes, communication pour le développement. 